\title{
On the Reconstruction Theorem of Holonomic Modules in the Gevrey Classes
}

By

\author{
Naofumi HondA*
}

§o. Introduction

The notion of holonomic systems entails a natural generalization of ordinary differential equations to higher dimension. A holonomic system is, by definition, a left coherent $\mathcal{E}$ (or $\mathscr{D}$ ) module whose characteristic variety is Lagrangian. It enjoys many good properties (see Kashiwara [K1], [K2] and Kashiwara-Kawai $[\mathrm{K}-\mathrm{K}]$ ): for example, all cohomology groups associated with its solution sheaf are constructible. As special functions satisfy systems of ordinary differential equations with regular singularities, a holonomic system with regular singularities introduced by Kashiwara-Oshima $[\mathrm{K}-\mathrm{O}]$ and $[\mathrm{K}-\mathrm{K}]$ is particularly important. It is well known that the category of holonomic modules with regular singularities is equivalent to that of perverse sheaves through Riemann-Hilbert correspondence (Kashiwara [K3] and Mebkout [Me]). Moreover the regularity of holonomic modules are stable under many operations (integration, restriction, etc.). For holonomic modules with irregular singularities, Kashiwara-Kawai obtained the following remarkable theorem.

Theorem ([K-K; Theorem 5.2.1]). Let $X$ be a complex manifold and $\mathscr{M}$ a holonomic $\mathcal{E}_{X}$ module. Then there exists a holonomic $\mathcal{E}_{X}$ module $\mathcal{M}_{\text {reg }}$ with regular singularities such that

$$
\mathcal{E}_{X}^{\infty} \otimes_{\varepsilon_{X}} \mathscr{M} \simeq \mathcal{E}_{X}^{\infty} \otimes_{\varepsilon_{X}} \mathscr{M}_{\text {reg }}
$$

This implies that all holonomic modules are transformed into holonomic modules with regular singularities by use of micro-differential operators of in-

Communicated by M. Kashiwara, December 3, 1990.

1991 Mathematics Subject Classification: 35N10, 58G07, 46P99

* University of Hokkaido, Faculty of Science, Department of Mathematics, Sapporo, 060, Japan 
finite order. In this paper, we show that this transformation can be achieved by a smaller class of operators (micro-differential operators of Gevrey growth order) corresponding to irregularity of modules.

Maim Theorem. Let $\mathscr{M}$ be a holonomic $\mathcal{E}_{X}$ module with irregularity at most $\sigma$. Then there exists a holonomic $\mathcal{E}_{X}$ module $\mathscr{M}_{\text {reg }}$ with regular singularities such that for all $s \in\left[1, \frac{\sigma}{\sigma-1}\right]$

$$
\mathcal{E}_{X}^{(s)} \otimes_{\varepsilon_{X}} \mathscr{M} \simeq \mathcal{E}_{X}^{(s)} \otimes_{\varepsilon_{X}} \mathscr{M}_{\text {reg }} .
$$

Refer to Section 1 and Section 2 for the definitions of irregularity and the sheaf $\mathcal{E}_{X}^{(s)}$. Remark that restricting this result to the zero section, we obtain the same result for holonomic $\mathscr{D}_{X}$ modules.

The plan of our paper is as follows. In Section 1, we prepare some notation and give a review of operators of Gevrey class and also of regular singularities of modules. In Section 2, we define irregularity of modules. In Section 3 , we first review holonomic modules of $D$ type, and study multi-valued holomorphic functions of Gevrey growth order. Section 4 and 5 will be devoted to the proof of the main theorem.

The author would like to express his gratitude to Prof. N. Tose for valuable advice. $\mathrm{He}$ is also grateful to Prof. $\mathbb{H}$. Komatsu for encouragement.

\section{$\S$ 1. Preliminary}

\subsection{Micro-differential operators and holomorphic microfunctions of Gevrey} growth order。

We recall the definition of micro-differential operators of Gevrey growth order (refer to Aoki [A 1] for details).

Let $X$ be a complex manifold of dimension $n$ and $\pi: T^{*} X \rightarrow X$ its cotangent bundle. Set $\stackrel{\circ}{T}^{*} X=T^{*} X \backslash T_{X}^{*} X$ and denote by $\frac{\circ}{\pi}$ the restriction of $\pi$ to $\stackrel{\circ}{ }^{*} X$. We choose a local coordinate system of $X$ as $\left(x_{1}, \cdots, x_{n}\right)$ and that of $T^{*} X$ as $\left(x_{1}, \cdots, x_{n} ; \xi_{1}, \cdots, \xi_{n}\right) . \quad T^{*} X$ is endowed with the sheaf $\mathcal{E}_{X}^{\infty}$ of microdifferential operators of infinite order constructed by Sato-Kashiwara-Kawai $[\mathrm{S}-\mathbb{K}-\mathbb{K}]$.

Definition $\mathbb{1}_{\text {.1 }}$. For an open subset $U$ of $T^{*} X$, a formal sum $\sum_{i \in Z} P_{i}(x, \xi)$ belongs to $\mathcal{E}_{X}^{\infty}(U)$ if and only if the following conditions (1) and (2) are satisfied.

(1) $P_{i}(x, \xi)$ is holomorphic on $U$ and homogeneous of order $i$ with respect 
to $\xi$.

(2) For any compact set $K$ of $U$ and any positive real number $\varepsilon$, there exists a positive constant $C_{\varepsilon, K}$, and for any compact set $K$, there exists a positive constant $C_{K}$ such that

$$
\begin{aligned}
& \sup _{K}\left|P_{i}(x, \xi)\right| \leq C_{\varepsilon, K} \frac{\varepsilon^{i}}{i !} \quad(i \geq 0), \\
& \sup _{K}\left|P_{-i}(x, \xi)\right| \leq C_{K}^{i} i ! \quad(i \geq 0) .
\end{aligned}
$$

We denote by $\mathcal{E}_{X}$ (resp. $\mathcal{E}_{X}(m)$ ) the subsheaf of $\mathcal{E}_{X}^{\infty}$ consisting of microdifferential operators of finite order (resp. micro-differential operators of order at most $m$ ). For the theory of $\varepsilon_{X}$, refer to [S-K-K] and Schapira [S]. Now we define the subsheaf $\mathcal{E}_{X}^{(s)}$ of micro-differential operators of Gevrey growth order $(s)$ for any $s \in(1, \infty)$.

Definition 1.2. For an open subset $U$ of $T^{*} X$, a sum $\sum_{i \in Z} P_{i}(x, \xi) \in \mathcal{E}_{X}^{\infty}(U)$ belongs to $\mathcal{E}_{X}^{(s)}(U)$ if and only if $\left\{P_{i}\right\}_{i \in N}$ satisfies the following estimate (1.3) instead of (1.1); for any compact set $K$ of $U$, there exists a positive constant $C_{K}$ such that

$$
\sup _{K}\left|P_{i}(x, \xi)\right| \leq \frac{C_{K}^{i}}{i !^{s}} \quad(i \geq 0) \text {. }
$$

For convenience, we set $\mathcal{E}_{X}^{(1)}:=\mathcal{E}_{X}^{\infty}$ and $\mathcal{E}_{X}^{(\infty)}:=\mathcal{E}_{X}$. Moreover restricting the sheaf $\mathcal{E}_{X}^{(s)}$ to $T_{X}^{*} X$, we obtain the sheaf $\mathscr{D}_{X}^{(s)}$ on $X$. $\mathscr{D}_{X}^{(s)}$ is nothing but the sheaf of differential operators of Gevrey growth order $(s)$.

Next we review briefly the definition of the sheaf of holomorphic microfunctions. Let $Y$ be a complex submanifold of $X$ and $T_{Y}^{*} X$ its conormal bundle. We choose a local coordinate system $\left(x^{\prime}, x^{\prime \prime}\right)$ of $X$ so that $Y=\left\{x^{\prime \prime}=0\right\}$, and then $\left(x^{\prime} ; \xi^{\prime \prime}\right)$ is the corresponding local coordinate system of $T_{Y}^{*} X$. A section of the sheaf $\mathcal{C}_{Y \mid X}^{(s)}(s \in(1, \infty))$ of holomorphic microfunctions of Gevrey growth order $(s)$ on $T_{Y}^{*} X$ is a formal sum $\sum_{i \in Z} P_{i}\left(x^{\prime}, \xi^{\prime \prime}\right)$ which satisfies conditions (1.2) and (1.3). If $s=1$, we replace the estimate (1.3) to (1.1) and denote it by $\mathcal{C}_{Y \mid X}^{\infty}$. The sheaf $\mathcal{C}_{Y \mid X}^{(\infty)}$ is the subsheaf of $\mathcal{C}_{Y \mid X}^{\infty}$ consisting of sums $\sum_{i \in Z} P_{i}$ with $P_{i} \equiv 0$ for $i \gg 0$ and denoted by $\mathcal{C}_{Y \mid X}$. Remark that $\mathcal{C}_{Y \mid X}^{(s)}$ is an $\mathcal{E}_{X}^{\left(s^{\prime}\right)}$ mudule for any $s^{\prime} \in[s, \infty]$.

It is known that (cf. [S-K-K])

$$
\mathcal{E}_{X}^{(s)}=\mathcal{C}_{X \mid X \times X}^{(s)} \otimes \Omega_{X}
$$

and 


$$
\mathcal{C}_{Y \mid X}^{(s)}=\mathcal{E}_{X}^{(s)} \otimes_{\varepsilon_{X}} \mathcal{C}_{Y \mid X}
$$

Here we denote by $\Omega_{X}$ the sheaf of holomorphic $n$ forms. In Section 4 , the sheaf $\mathcal{C}_{Y \mid X}^{R}$ on $T_{Y}^{*} X$ plays an important role. It is defined by

$$
\mathcal{C}_{Y \mid X}^{R}:=\mu_{Y}\left(\mathcal{O}_{X}\right)[\operatorname{codim} Y]
$$

where $\mu_{Y}\left({ }^{\circ}\right)$ is Sato's microlocalization functor (for its definition and properties, see $\mathbb{K}$ ashiwara-Schapira $[\mathbb{K}-\mathbb{S} 1]$ and $[\mathbb{K}-\mathbb{S} 2])$.

\subsection{Definition of regular singularities for coherent modulles.}

The notion of regular singularities for modules has been introduced and studied in $[\mathbb{K}-0]$ and $[\mathbb{K}-\mathbb{K}]$. We recall its definition and make several important remarks. Let $V$ be an involutive analytic subset of $\stackrel{\circ}{T}^{*} X$ and $p \in \stackrel{\circ}{T}^{*} X$. Kashiwara-Oshima introduced the Noetherian subring $\mathcal{E}_{V}$ of $\mathcal{E}_{X}$ to define regular singularities. For the definition of $\mathcal{E}_{\mathrm{V}}$, refer to $[\mathrm{K}-\mathrm{O}]$ and see Section 2 in the case that $V$ is smooth.

Definition 1.30 ([K-O]). A coherent $\mathcal{E}_{X}$ module $\mathscr{M}$ has regular singularities at $p$ along $V$ if and only if the following equivalent conditions are satisfied.

(1) There are a neighborhood $U$ of $p$ and an $\mathcal{E}_{V}$ module $\mathscr{H}_{0}$ of $\mathscr{M}$ defined on $U$ which is coherent over $\mathcal{E}_{X}(0)$ and generates $\mathcal{M}$ as an $\mathcal{E}_{X}$ module.

(2) Any coherent $\mathcal{E}_{V}$ submodule of $\mathscr{M}$ defined in a neighborhood of $p$ is coherent over $\mathcal{E}_{X}(0)$.

We say a holonomic $\mathcal{E}_{X}$ module $\mathscr{M}$ on $U\left(\subset \stackrel{\circ}{T}^{*} X\right)$ has regular singularities if it has regular singularities along supp $\mathscr{H}$ at all points $p \in U$.

On the other hand, Kashiwara-Kawai introduced the notion of $\mathbb{R}$.S., seemingly different from regular singularities given in the above definition.

Definition $\mathbb{1}_{0} 4([\mathbb{K}-\mathrm{K}])$. A holonomic $\mathcal{E}_{X}$ module $\mathscr{M}$ on $U\left(\subset \dot{T}^{*} X\right)$ has $R$.S. if it has regular singularities at all points in an open dense subset of $U$.

One of the deepest results of $[\mathbb{K}-\mathrm{K}]$ is the fact that two regular singularity conditions in Definition 1.3 and 1.4 are actually equivalent for holonomic modules. The notion of irregularity at most (1), to be introduced in the next section, also coincides with them under the condition of holonomicity.

\section{$\S 2$. The Sheaf $\mathcal{E}_{V}^{(\sigma)}$ amd Irregullarity of Modules}

The aim of this section is to define irregularity of coherent $\varepsilon_{X}$ modules 
and investigate its properties. Although there exist several definitions of irregularity (cf. [A2], [Ko1], [L] and [M1]), we give, in this paper, its definition using the sheaf $\mathcal{E}_{(V)}^{(\sigma)}$.

Let $X$ be a complex manifold, $\pi: T^{*} X \rightarrow X$ its cotangent bundle, and $V$ a regular or maximally degenerate involutive submanifold of codimension $d \geq 1$ in $i^{*} X$. To define the sheaf $\left.\mathcal{E}_{(v)}^{(\sigma)}\right)$, we introduce the following notion.

Defimition 2.1. We say micro-differential operators $\left(P_{1}, \cdots, P_{d}\right)$ are involutive coordinate operators of $V$ at $p \in V$ if and only if

(1) all $P_{i}$ 's are strictly of order one (i.e. $P_{i} \in \mathcal{E}_{X}(1), \notin \mathcal{E}_{X}(0)$ ),

(2) $\left[P_{i}, P_{j}\right]=0$ for all $1 \leq i, j \leq d$,

(3) the principal symbols $\left(\sigma\left(P_{1}\right), \cdots, \sigma\left(P_{d}\right)\right)$ form a base of the defining ideal $I_{V}$ of $V$ in a neighborhood of $p$.

Remark that involutive coordinate operators of $V$ always exist.

Now we define the sheaf of rings $\mathcal{E}_{(\mathrm{V})}^{(\sigma)}$ on $\stackrel{\circ}{T}^{*} X$ for a rational number $\sigma \in[1, \infty)$. In case $\sigma=1$, this sheaf coincides with the sheaf $\mathcal{E}_{V}$ defined in [K-O] and $[\mathrm{K}-\mathrm{K}]$.

Definition 2.2. A sheaf $\mathcal{E}_{(V)}^{(\sigma)}$ is defined in the following way.

(1) On the outside of $V,\left.\mathcal{E}_{(V)}^{(\sigma)}\right|_{T^{\circ} * X \backslash V}:=\left.\mathcal{E}_{X}\right|_{T^{\circ} * X \backslash V}$.

(2) On $V$, a micro-differential operator $P$ belongs to $\mathcal{E}_{(V)}^{(\sigma)}$ at $p \in V$ if and only if there exist involutive coordinate operators $\left(P_{1}, \cdots, P_{d}\right)$ at $p$ satisfying

$$
P \in \sum_{0 \leq \alpha_{i}} \varepsilon_{X}(l(\alpha)) P_{1}^{\alpha} \ldots P_{d^{d}}^{\alpha} \text { at } p
$$

Here $l(\alpha)$ is the largest integer satisfying $l \leq \frac{1-\sigma}{\sigma}|\alpha|$.

Remark that this definition does not depend on the choice of involutive coordinate operators. In fact, if $\left(\widetilde{P}_{1}, \cdots, \widetilde{P}_{d}\right)$ be other involutive coordinate operators of $V$ at $p$, then there exist micro-differential operators of order zero $Q_{i, j}, R_{i}(1 \leq i, j \leq d)$ such that

$$
P_{i}=\sum_{j=1}^{d} Q_{i, j} \widetilde{P}_{j}+R_{i}
$$

Then it is easy to see for $\left(\widetilde{P}_{1}, \cdots, \widetilde{P}_{d}\right),(2.1)$ holds.

From this observation, we find that $\mathcal{E}_{(V)}^{(\sigma)}$ is stable under quantized contact transformations. We list up the main properties of the sheaf $\mathcal{E}\left(\begin{array}{l}(\sigma) \\ V\end{array}\right)$. 
(1) $\mathcal{E}_{V}^{(\sigma)}$ is a subring of $\mathcal{E}_{X}$.

(2) $\mathcal{E}_{X}(0) \subset \mathcal{E}_{(V)}^{(\sigma)}$, and $\mathcal{E}_{(V)}^{(\sigma)}$ is a left and right $\mathcal{E}_{X}(0)$ module.

(3) $\mathcal{E}_{(V)}^{(\sigma)}$ is a sheaf of Noetherian ring, and any coherent $\mathcal{E}_{X}$ module is pseudo-coherent over $\mathcal{E}_{(V)}^{(\sigma)}$.

(4) If $P \in \mathcal{E}_{(V)}^{(\sigma)}$, then its formal ajoint operator $P^{*}$ belongs to $\mathcal{E}_{(V)}^{(\sigma)}$. Since the proof of (1)-(4) is the same as [K-K; Chapter 1], we omit it. For a holomorphic function $f \in \mathcal{O}_{T^{*} X \text {, }}$, we denote the vanishing order of $f$ along $V$ at $p$ by $m_{(v), p}(f)$ (i.e. $m_{(v), p}(f)=k$ if and only if $f \in I_{V}^{k}$ and $f \notin I_{V}^{k+1}$ ).

Example 2.3. Let $X=\mathbb{C}^{n}$ with a coordinate system $\left(x_{1}, \cdots, x_{n}\right), V=$ $\left\{\left(x_{1}, \cdots, x_{n} ; \xi_{1}, \cdots, \xi_{n}\right) \in \stackrel{\circ}{T}^{*} X ; x_{1}=\xi_{2}=\cdots=\xi_{r}=0, \xi_{1} \neq 0\right\}$ and $p=\left(0 ; d x_{1}\right)$. In this case, we may choose $\left(x_{1} \partial_{1}, \partial_{2}, \cdots, \partial_{r}\right)$ as involutive coordinate operators, and Definition 2.2 is equivalent to the following condition with the above coordinates; $P \in \mathcal{E}_{X}(m)$ belongs to $\mathcal{E}_{(V)}^{(\sigma)}$ at $p$ if and only if the symbol expansion $P(x, \xi)=P_{m}(x, \xi)+P_{m-1}(x, \xi)+\cdots$ of $P(x, D)$ satisfies the condition

$$
m_{(v), p}\left(P_{i}(x, \xi)\right) \geq \sigma i \quad(i \leq m) .
$$

Moreover if $\sigma=\frac{q}{p}$ with $q \geq p$ positive integers and prime to each other, we have

$$
\mathcal{E}_{(V)}^{(\sigma)}=\mathcal{E}_{X}(0)\left[\left(x_{1}^{\beta} \partial_{1}^{\alpha} \partial_{2}^{\beta} \cdots \partial_{r}^{\beta}\right)\right]\left(\begin{array}{l}
1 \leq k \leq p, \alpha \in \mathbb{Z} \\
\beta_{1} \geq 0, \cdots, \beta_{r} \geq 0, \\
\alpha+\beta_{2}+\cdots+\beta_{r}=k, \\
\beta_{1}+\beta_{2}+\cdots+\beta_{r}=s(k)
\end{array}\right) .
$$

Here $s(k)$ is the smallest integer satisfying $s \geq \sigma k$.

Let $V$ be a regular or maximally degenerate involutive subvariety in $\stackrel{\circ}{T}^{*} X$ and $p \in \stackrel{\circ}{T}^{*} X$. We first define irregularity of a coherent $\mathcal{E}_{X}$ module in case $V$ is smooth. Form now on, we always assume $\sigma(\in[1, \infty))$ is a rational number.

Definition 2.4. A coherent $\mathcal{E}_{X}$ module $\mathscr{M}$ has irregularity at most $\sigma$ along $V$ at $p$ if and only if $\mathscr{M}$ satisfies one of the following equivalent conditions.

(1) There exist an open neighborhood $U$ of $p$ and a coherent $\mathcal{E}_{X}(0)$ module $\mathscr{M}_{0}$ on $U$ which is an $\mathcal{E}_{(V)}^{(\sigma)}$ module and generates $\mathscr{M}$ over $\mathcal{E}_{X}$.

(2) Any coherent $\mathcal{E}_{(V)}^{(\sigma)}$ submodule of $\mathscr{M}$ in a neighborhood of $p$ is a coherent $\mathcal{E}_{X}(0)$ module.

Next we define irregularity in the general case. Donote by $V_{r e g}$ the smooth 
part of $V$.

Definition 2.5. (i) $A$ coherent $\mathcal{E}_{X}$ module $\mathscr{M}$ has irregularity at most $\sigma$ along $V$ at $p$ if and only if for some open neighborhood $U$ of $p . \mathscr{M}$ has irregularity at most $\sigma$ along $V_{\text {reg }}$ at any point $\tilde{p} \in V_{\text {reg }} \cap U$.

(ii) $A$ holonomic $\mathcal{E}_{X}$ module $\mathscr{M}$ has irregularity at most $\sigma$ if and only if it has irregularity at most $\sigma$ along supp $(\mathscr{M})$ on the outside of $T_{X}^{*} X$.

(iii) $A$ holonomic $\mathscr{D}_{X}$ module $\mathscr{\Omega}$ has irregularity at most $\sigma$ if and only if $\mathcal{E}_{X} \otimes \mathscr{D}_{X} \Re$ has irregularity at most $\sigma$.

We give several lemmas about properties of irregularity.

Lemma 2.6. Let $V$ be a regular or maximally degenerate involutive submanifold of $\stackrel{\circ}{T}^{*} X$. Assume a coherent $\mathcal{E}_{X}$ module $\mathscr{M}$ has irregularity at most $\sigma$ along $V$. Then $\operatorname{supp}(\mathscr{M}) \subset V$.

Lemma 2.7. Let $0 \rightarrow \mathscr{M}_{1} \rightarrow \mathscr{M} \rightarrow \mathscr{M}_{2} \rightarrow 0$ be an exact sequence of coherent $\mathcal{E}_{X}$ modules. Then $\mathscr{M}$ has irregularity at most $\sigma$ along $V$ if and only if $\mathscr{M}_{1}$ and $\mathscr{M}_{2}$ have irregularity at most $\sigma$ along $V$.

The above two lemmas can be proved in the same way as [K-K; Lemma 1.1.13 and Lemma 1.1.14].

Lemma 2.8. Let $V \subset V_{1}$ be regular or maximally degenerate involutive submanifolds of $\stackrel{\circ}{T}^{*} X$. If a coherent $\mathcal{E}_{X}$ module $\mathscr{M}$ has irregularity at most $\sigma$ along $V$, then it has irregularity at most $\sigma$ along $V_{1}$.

Proof. Since $\mathcal{E}_{\left(V_{1}\right)}^{(\sigma)} \subset \mathcal{E}_{(V)}^{(\sigma)}$, it is clear. 竵

We give two examples.

Example 2.9.

(1) Let $X=C^{n}$ with a coordinate system $\left(x_{1}, \cdots, x_{n}\right), V=\left\{\left(x_{1}, \cdots, x_{n}\right.\right.$; $\left.\left.\xi_{1}, \cdots, \xi_{n}\right) \in \stackrel{\circ}{T}^{*} X ; \xi_{1}=0, \xi_{n} \neq 0\right\}$ and $p=\left(0 ; d x_{n}\right)$. Let $P(x, D)=D_{1}^{m}+P_{m-1}\left(x, D^{\prime}\right)$ $D_{1}^{m-1}+\ldots+P_{0}\left(x, D^{\prime}\right)$ be a micro-differential operator of Weierstrass type with respect to $D_{1}$ satisfying $\operatorname{ord}\left(P_{k}\left(x, \xi^{\prime}\right)\right)<m-k(0 \leq k \leq m-1)$. We define a rational number $\operatorname{Irr}_{V, p}(P)$ by

$$
\operatorname{Irr}_{V, p}(P)=\max \left\{1, \frac{m-k}{m-k-\operatorname{ord}\left(P_{k}\left(x, \xi^{\prime}\right)\right)}\right\} .
$$


Then it is easy to see an $\mathcal{E}_{X}$ coherent module $\frac{\mathcal{E}_{X}}{\mathcal{E}_{X} P}$ has irregularity at most $\operatorname{Irr}_{V, p}(P)$ along $V$. Conversely we obtain the following lemma.

Lemma 2.10. Let $M$ be a coherent $\mathcal{E}_{X}$ module which has irregularity at most $\sigma$ along $V$ at $p$. Then for any $u \in \mathcal{M}$, there exists a micro-differential operator $\mathbb{P}$ of Weierstrass type with respect to $D_{1}$ such that $\mathbb{P} u=0$ and $\operatorname{Irr}_{V, p}$ $(P) \leq \sigma$.

Proof. We may assume $\mathscr{M}=\mathscr{D}_{X} u$ and $\sigma=\frac{q}{p}$ with $q \geq p$ positive integers and prime to each other. Then $\mathscr{M}_{0}:=\mathcal{E}_{(V)}^{(\sigma)} u$ is a coherent $\mathcal{E}_{X}(0)$ module, and $\theta:=D_{1}^{q} D_{n}^{p-q}$ belongs to $\mathcal{E}_{(V)}^{(\sigma)}$. Thus the increasing sequence $\mathscr{M}_{k}=\sum_{j=0}^{k} \mathcal{E}_{X}(0) \theta^{j} u$ is locally stationary, and we can find an operator $Q=\theta^{m}+Q_{m-1}(x, D) \theta^{m-1}+\cdots+$ $Q_{0}(x, D)$ with $Q_{i}(x, D) \in \mathcal{E}_{X}(0)$ such that $Q u=0$. Finally we apply the Weierstrass division theorem to the operator $Q$. 国

(2) Let $X=\mathbb{C}, V=T_{(0)}^{*} X=\{(x ; \xi) ; x=0, \xi \neq 0\}, q=0$ and $p=(0 ; d x)$. Let $P(x, D)=x^{d} D^{m}+a_{m-1}(x) D^{m-1}+\cdots+a_{0}(x)$ be a differential operator of order $m$. In the same way as the above example, we define a rational number $\operatorname{Irr}_{V, p}(P)$ by

$$
\operatorname{Irr}_{V, p}(P)=\max \left\{1, \frac{d-m_{\{q\}}\left(a_{k}(x)\right)}{m-k}\right\}
$$

Then an $\mathcal{E}_{X}$ coherent module $\frac{\mathcal{E}_{X}}{\mathcal{E}_{X} P}$ has irregularity at most $\operatorname{Irr}_{V, p}(P)$ along $V$.

Lemma 2.11. Let $\mathcal{M}$ be a coherent $\mathcal{E}_{X}$ module which has irregularity at most $\sigma$ along $V$ at $p$. Then for any $u \in \mathscr{M}$, there exists a differential operator $\mathbb{P}$ such that $P u=0$ and $\operatorname{Irr}_{V, p}(\mathbb{P}) \leq \sigma . \quad$ (Remark that although $\mathscr{M}$ is an $\mathcal{E}_{X}$ module, we can find such an operator in the category of differential operators.)

Proof. Since $\mathscr{M}$ is holonomic, a coherent $\mathcal{E}_{X}(0)$ module $\mathcal{E}_{(V)}^{(\sigma)} u$ is a finitely generated $\mathcal{O}_{q}$ module by the result of Malgrange [M2] and Björk [B]. Thus employing the same argument as Lemma 2.10, we can find a desired differential operator $P$. 图

At the end of this section, we investigate the functoriality of irregularity by the inverse image and the dual. Let $f: Y \hookrightarrow X$ be an inclusion of two complex manifolds. We associate the morphisms $\bar{\omega}$ and $\rho$ as usual;

$$
T^{*} Y \stackrel{\rho}{\leftarrow} T^{*} X \times_{Y} Y \stackrel{\widetilde{\omega}}{\rightarrow} T^{*} X
$$


Lemma 1.12. Let $V$ be a regular or maximally degenerate involutive submanifold of $\stackrel{\circ}{*}^{*} X$ and $\mathscr{M}$ a coherent $\mathcal{E}_{X}$ module which has irregularity at most $\sigma$ along $V$. If $V$ and $Y$ are symplectically orthogonal, then $\mathscr{M}_{Y}=\rho_{*}\left(\mathcal{E}_{Y \rightarrow X} \otimes_{\bar{\omega}}-1 \mathcal{E}_{X}\right.$ $\left.\bar{\omega}^{-1} \mathscr{M}\right)$ has irregularity at most $\sigma$ along $\rho \bar{\omega}^{-1}(V)$.

Lemma 1.13. Let $V$ be a regular or maximally degenerate involutive submanifold of $\stackrel{\circ}{*}^{*} X$ and $\mathscr{M}$ a coherent $\mathcal{E}_{X}$ module with irregularity at most $\sigma$ along $V$. Then all cohomology groups of $\mathbb{R} H a m \mathcal{E}_{X}\left(\mathscr{H}, \mathcal{E}_{X}\right) \otimes \Omega_{X}^{-1}$ are coherent $\mathcal{E}_{X}$ modules with irregularity at most $\sigma$ along $V$.

Since the proofs of above two lemmas are easy, we leave them to the readers.

\section{§ 3. Multi-valued Holomorphic Functions of Gevrey Growth Order}

In this section, we investigate the properties of multi-valued holomorphic functions of Gevrey growth order which we need in the next section. First we briefly recall the definition of Nilsson class functions and that of Holonomic $D$ type modules. For details, refer to [K-K; Chapter 2].

Let $X$ be a complex manifold of dimension $n, S$ a complex hypersurface of $X, L$ a local system over $C$ on $X \backslash S$ with rank $m$ and $j: X \backslash S \hookrightarrow X$ an open inclusion.

A holonomic $\mathscr{D}_{X}$ module of $D$ type $\mathcal{L}\left(\subset j_{*}\left(\mathcal{O}_{X \backslash S} \otimes_{C} L\right)\right)$ is characterized by the following three conditions (1), (2) and (3).

(1) $\operatorname{char}(\mathcal{L}) \subset T_{X}^{*} X \cup \pi^{-1}(S)$.

(2) $\mathcal{L}$ is a holonomic $\mathscr{D}_{X}$ module with R.S.

(3) $\mathcal{H}_{[S]}^{i}(\mathcal{L})=0$ for any $i$.

We give, however, more explicit expressions for holonomic systems of $D$ type. Fix a nonsingular point $q \in S$ and choose a local coordinate system $\left(x_{1}, x^{\prime}\right)=\left(x_{1}, \cdots, x_{n}\right)$ in a neighborhood $U$ of $q$ so that $q=0$ and that $U \cap S$ is defined by $x_{1}=0$. Set $U^{ \pm}=\left\{x \in U ; \pm \Re x_{1}>-\left|\Im x_{1}\right|\right\}$. Then $L$ is a constant sheaf on $U^{ \pm}$and we have the isomorphism $f_{ \pm}:\left.L\right|_{U_{ \pm}} \rightarrow C_{U_{ \pm}}^{m}$. Then the morphism $f_{ \pm, i}(1 \leq i \leq m)$ denotes the composition of $f_{ \pm}$and the $i$-th projection: $\boldsymbol{C}^{m} \rightarrow \boldsymbol{C}$.

Definition 3.1. (i) A section $u \in j_{*}\left(\Theta_{X \backslash S} \otimes_{C} L\right)$ at $q$ belongs to the Nilsson class at $q$ if and only if there exist positive constants $l, C$ and $\varepsilon$ with the estimate

$$
\left|f_{ \pm, i}(u)(x)\right| \leq C\left|x_{1}\right|^{-l}
$$


for $x \in U^{ \pm}$and $|x|<\varepsilon$.

(ii) The subsheaf $\mathcal{L}_{n i l}$ is defined in such a way that for an open subset $U$, $\mathcal{L}_{n i l}(U)=\left\{u \in j_{*}\left(\mathcal{O}_{X \backslash S} \otimes_{C} L\right)(U) ; u\right.$ belongs to the Nilsson class at all points $\left.q \in S_{\text {reg }} \cap U\right\}$.

The fact that $\mathcal{L}=\mathcal{L}_{n i l}$ was proved in $[\mathrm{K}-\mathbb{K}$; Chapter 2]. Moreover the following theorem was also obtained.

Theorem 3.2. ([K-K; Theorem 2.2.4]). There exists a $\mathscr{D}_{X}^{\infty}$ isomorphism

$$
\Phi: \mathscr{D}_{X}^{\infty} \otimes_{\mathscr{D}_{X}} \mathcal{L} \simeq j_{*}\left(\Theta_{X \backslash S} \otimes_{C} L\right)
$$

Since the ring $\mathscr{D}_{X}^{\infty}$ is faithfully flat over the ring $\mathscr{D}_{X}^{(s)}(s \in[1, \infty]), \mathcal{L}^{(s)}:=$ $\mathscr{D}_{X}^{(s)} \otimes \mathscr{D}_{X} \mathcal{L}$ is considered as a submodule of $\mathcal{L}^{\infty}:=\mathscr{D}_{X}^{\infty} \otimes \mathscr{D}_{X} \mathcal{L}$. Then we have the following lemma.

Lemma 3.3. We have a $\mathscr{D}_{X}^{(s)}$ isomorphism

$$
\Phi: \mathscr{D}_{X}^{(s)} \otimes_{\mathscr{D}_{X}} \mathcal{L} \simeq \mathscr{D}_{X}^{(s)} \mathcal{L} \quad\left(\subset j_{*}\left(\mathcal{O}_{X \backslash s} \otimes_{C} L\right)\right)
$$

By (3.3), we identify $\mathscr{D}_{X}^{(s)} \otimes_{\mathscr{D}_{X}} \mathcal{L}$ with $\mathscr{D}_{X}^{(s)} \mathcal{L}$ in $j_{*}\left(\mathcal{O}_{X \backslash S} \otimes_{C} L\right)$.

From now on, we assume $S$ is a smooth complex hypersurface. Let $\gamma$ be a generator of the fundamental group $\pi_{1}(X \backslash S)$ and $\operatorname{Exp}(-2 \pi i L) \in G L(m, \mathbb{C})$ a monodoromy matrix of the local system $L$ with respect to $r$. We may assume the matrix $L$ is upper diagonal and its eigenvalues $\notin \mathbb{N}$. Now we define the subsheaf of $j_{*}\left(\mathcal{O}_{X \backslash S} \otimes_{C} L\right)$ consisting of multi-valued holomorphic functions of Gevrey growth order. Assume $s \in(1, \infty)$.

Definition 3.4. (i) A section $u \in j_{*}\left(\Theta_{X \backslash S} \otimes_{C} L\right)_{q}$ belongs to the Gevrey Nilsson class of order $(s)$ at $q \in S$ if and only if there exist positive constants $l, C$ and $\varepsilon$ satisfying the estimate

$$
\left|f_{ \pm, i}(u)(x)\right| \leq C \exp \left(\frac{l}{\left|x_{1}\right|}\right)^{1 /(s-1)}
$$

for $x \in U^{ \pm}$and $|x|<\varepsilon$.

(ii) We define the subsheaf $\mathcal{L}_{n i l}^{(s)}$ by assigning $\mathcal{L}_{n i l}^{(s)}(U)=\left\{u \in j_{*}\left(\mathcal{O}_{X \backslash S} \otimes_{C} L\right)(U) ; u\right.$ belongs to the Gevrey Nilsson class of $\operatorname{order}(s)$ at all points $q \in S \cap U\}$.

It is easy to see that $\mathcal{L}_{n i l}^{(s)}$ is a $\mathscr{D}_{X}^{(s)}$ module. We need the following proposition in the next section which clarifies the relations between $\mathcal{L}_{n i l}^{(s)}$ and $\mathcal{L}_{n i l}$. 
Proposition 3.5. In the above situation, we have

$$
\mathcal{L}_{n i l}^{(s)}=\mathscr{D}_{X}^{(s)} \otimes \mathscr{D}_{X} \mathcal{L}_{n i l}
$$

To prove this proposition, we prepare some notation. We take a local coordinate system $\left(x_{1}, x^{\prime}\right)=\left(x_{1}, x_{2}, \cdots, x_{n}\right)$ of $X$ so that $S=\left\{x_{1}=0\right\}$ and $q=0$. Set

$O^{(s)}:=\left\{f \in\left(j_{*} \mathcal{O}_{X \backslash S}\right)_{q}\right.$; there exist a neighborhood $U$ of $q$ and constants $l, C$ so that the function $f$ satisfies the estimate

$$
|f(x)| \leq C \operatorname{Exp}\left(\frac{l}{\left|x_{1}\right|}\right)^{1 / s-1}
$$

for $x \in U \backslash S\}$.

Remark that $f \in O^{(s)}$ if and only if there exist an open disk $U$ centered at 0 , positive constants $C$ and $l$, the Laurent expansion of $f$ with respect to $x_{1}$ on $U$

$$
f(x)=f_{0}(x)+\sum_{\alpha>0} f_{\alpha}\left(x^{\prime}\right) x_{1}^{-\infty}
$$

with $f_{0}(x) \in \mathcal{O}(U)$ satisfying the estimate

$$
\left|f_{\alpha}\left(x^{\prime}\right)\right| \leq C l^{\alpha}\left(\frac{1}{\alpha !}\right)^{s-1}
$$

To reduce the local system $L$ to be trivial, we consider a $C$ linear morphism

$$
\begin{aligned}
& \Psi:\left(O^{(s)}\right)^{m} \longrightarrow j_{*}\left(\Theta_{X \backslash S} \otimes_{C} L\right)_{q} \\
& \vec{f} \quad \longrightarrow \operatorname{Exp}\left(\log \left(x_{1}\right) L\right) \vec{f} .
\end{aligned}
$$

This is apparently an injective $\boldsymbol{C}$ linear morphism (but not $\mathscr{D}_{X}$ linear). Moreover for $\vec{f} \in\left(O^{(s)}\right)^{m}$, we have

$$
\Psi\left(\left(\partial_{x_{1}}+\frac{L}{x_{1}}\right) \vec{f}\right)=\partial_{x_{1}} \Psi(\vec{f}) .
$$

Proof of Proposition 3.5. By Lemma 3.3, it is enough to show $\mathcal{L}_{n i l}^{(s)}=$ $\mathscr{D}_{X}^{(s)} \mathcal{L}_{n i l}$. Let $f(x) \in O^{(s)}$ and $\lambda_{j}$ be a $(j, j)$ component of the matrix $L$. The function $f$ has the Laurent expansion (3.6) to which we associate differential operators (of infinite order) $f^{(j)}(\tilde{D})$ and $f^{(j)}(D)$ as

$$
\begin{gathered}
f^{(j)}(\widetilde{D})=\sum_{\alpha \geq 0} f_{\alpha+1}\left(x^{\prime}\right)(-1)^{\alpha} \frac{\Gamma\left(-\lambda_{j}+1\right)}{\Gamma\left(-\lambda_{j}+\alpha+1\right)}\left(\partial_{x_{1}}+\frac{L}{x_{1}}\right)^{\alpha}, \\
f^{(j)}(D)=\sum_{\alpha \geq 0} f_{\alpha+1}\left(x^{\prime}\right)(-1)^{\alpha} \frac{\Gamma\left(-\lambda_{j}+1\right)}{\Gamma\left(-\lambda_{j}+\alpha+1\right)}\left(\partial_{x_{1}}\right)^{\alpha} .
\end{gathered}
$$


By direct calculations, we deduce the following facts.

(i) $f^{(j)}(D) \in \mathscr{D}_{X}^{(s)}$, and for any $\vec{g} \in\left(O^{(s)}\right)^{m}$

$$
\Psi\left(f^{(j)}(\widetilde{D}) \vec{g}\right)=f^{(j)}(D) \Psi(\vec{g})
$$

(ii)

$$
f^{(j)}(\tilde{D})\left(\begin{array}{c}
0 \\
\vdots \\
0 \\
\frac{1}{x} \\
0 \\
\vdots \\
0
\end{array}\right)=\left(\begin{array}{l}
h_{1}(x) \\
\vdots \\
h_{j-1}(x) \\
f(x)-f_{0}(x) \\
0 \\
\vdots \\
0
\end{array}\right)
$$

where $h_{1}(x), \cdots, h_{j-1}(x) \in O^{(s)}$.

Since $\vec{f}:=\Psi^{-1}(g)$ belongs to $\left(O^{(s)}\right)^{m}$ for any $g \in \mathcal{L}_{n_{2 l} l, q}^{(s)}$, we can find $u_{l} \in$ $\mathcal{L}_{n i l, q}$ and differential operators $P_{l} \in\left(\mathscr{D}_{X}^{(s)}\right)_{q}(1 \leq l \leq m)$ so that

$$
g=\sum_{l=1}^{m} P_{l} u_{l} \bmod \mathcal{L}_{n i l, q}
$$

This completes the proof. 囷

\section{$\S 4 . \quad$ Embedding of Holonomic Modules}

Let $X$ be a complex manifold of dimension $n+1$ and $Y$ a smooth complex hypersurface of $X$. Set $\Lambda=T_{Y}^{*} X \backslash T_{X}^{*} X, V=Y \times \stackrel{\circ}{T}^{*} X$ and $p \in \Lambda$, and fix a generator $r$ of the fundamental group $\pi_{1}(X \backslash Y)$. We prove the following embedding theorem which is analogous to [K-K; Theorem 4.1.1] in case of smooth $Y$.

Theorem 4.1. Let $\mathscr{M}$ be a holonomic $\mathcal{E}_{X}$ module at $p$ with $\operatorname{supp}(\mathscr{M}) \subset$ $\Lambda$. Assume $\mathscr{M}$ has irregularity at most $\sigma$ along $V$ at $p$. Then there exists a holonomic $\mathscr{D}_{X}$ module $\mathcal{L}$ with $R$.S. at $q=\pi(p)$ which enjoys the properties;

(1) for any $s \in\left[1, \frac{\sigma}{\sigma-1}\right]$, there exists an injective $\mathscr{D}_{X}^{(s)}$ linear morphism $E^{(s)}: \mathcal{E}_{X}^{(s)} \otimes \mathcal{E}_{X} \mathscr{M}_{p} \hookrightarrow \mathcal{L}_{q}^{(s)}$, and

(2) $E^{(s)}$ can be extended to an injective $\mathcal{E}_{X}^{(s)}$ morphism

$$
F^{(s)}: \mathcal{E}_{X}^{(s)} \otimes \mathcal{E}_{X} \mathscr{M}_{p} \hookrightarrow \mathcal{E}_{X}^{(s)} \otimes \mathcal{D}_{X}^{(s)} \mathcal{L}_{q}^{(s)}
$$


by $u \rightarrow 1 \otimes E^{(s)}(u)$.

Remarks. (1) $\mathcal{L}$ is isomorphic to the quotient of a holonomic $D$ type over a de Rham type module.

(2) The irregularity condition along $V$ is weaker than that along its support $\Lambda$.

To prove this theorem, we make full use of the technique of $[\mathrm{K}-\mathrm{K}]$. Since the problem is local, we may assume $X=C^{n+1}=\left(t, x_{1}, \cdots, x_{n}\right), Y=\{t=0\}, p=$ $(0 ; d t)$ and $q=0$. Let $B_{\varepsilon, x}$ (resp. $B_{\varepsilon, t}$ ) be open balls with radius $\varepsilon$ and center at 0 in $\mathbb{C}_{x}^{n}$ (resp. $\left.\mathbb{C}_{t}\right), B_{\varepsilon}=B_{\varepsilon, t} \times B_{\varepsilon, x}$ and $T_{\varepsilon}$ an open sector $\{t \in C ;|t|<\varepsilon, \varepsilon|\Im t|>$ Rt\}. Set $C:=\left(C_{Y \mid X}^{R}\right)_{p}$.

The $\left(\mathcal{E}_{X}^{\infty}\right)_{p}$ module $C$ plays an important role and has expression as the boundary values of holomorphic functions

$$
C=\underset{\varepsilon \rightarrow 0}{\lim } \frac{\mathcal{O}\left(T_{\varepsilon} \times B_{\varepsilon, x}\right)}{\mathcal{O}\left(B_{\varepsilon}\right)} .
$$

Let $\mathscr{M}$ be a holonomic $\mathcal{E}_{X}$ module at $p$. Since $H^{k} \mathbb{R} \operatorname{Ham}_{\mathcal{E}_{X}}\left(\mathscr{M}, \mathcal{C}_{Y \mid X}^{R}\right)$ is a constructible sheaf for any $k$, we obtain

$$
\operatorname{dim}_{C}\left(\operatorname{Ham}_{X}(\mathscr{M}, C)=\operatorname{Ham}_{x}^{\infty}\left(\mathscr{M}^{\infty}, C\right)\right)<\infty .
$$

Here we quote the following theorems of $[\mathrm{K}-\mathrm{K}]$.

Theorem 4.2 ([K-K; Theorem 4.5.2 and Theorem 4.5.3]). Let $\mathscr{M}$ be a holonomic $\mathcal{E}_{X}$ module with $\operatorname{supp}(\mathscr{M}) \subset \Lambda$. Let $\phi \in \operatorname{Hom}_{\mathcal{E}_{X}}(\mathcal{M}, C), u \in \mathscr{M}_{p}$ and $P \in \mathcal{E}_{X}^{\infty}$. Then a holomorphic representation $\widetilde{\phi}(P u)$ of $\phi(P u)$ can be extended as a multi-valued holomorphic function over $B_{\varepsilon} \backslash Y$ with finite determination for some $\varepsilon>0$.

From now on, we denote by $\tilde{\phi}(u)$ a holomorphic representation of $\phi(u)$. If $Y$ has singular points, the proof of this theorem is very difficult. In case $Y$ is smooth, however, we have an easier proof, which we give after the next proposition.

Before stating the next proposition, we make several preparations.

Let $\beta: \widetilde{X \backslash Y} \rightarrow X \backslash Y$ be a universal covering space. We define a class of multi-valued functions of growth order $(s)$. Set

$\tilde{O}^{(s)}=\lim _{\varepsilon \rightarrow 0}\left\{f \in \mathcal{O}\left(\beta^{-1}\left(B_{\varepsilon} \backslash Y\right)\right)\right.$; there exists $l>0$ such that for any branch of $f$ on $V_{\varepsilon}:=B_{\varepsilon} \backslash\left(\overline{\mathbb{R}^{+}} \times B_{\varepsilon, x}\right)$, we can find $C>0$ satisfying the estimate $|f(t, z)| \leq$ 
$C \operatorname{Exp}\left(\frac{l}{|t|}\right)^{1 /(s-1)}$ for $\left.(t, z) \in V_{\varepsilon}\right\}$.

Next we consider a restricted class of $\mathcal{E}_{X}^{(s)}$ which acts on the space $\tilde{O^{(s)}} / \mathcal{O}_{X, q}$. Set $\tilde{\mathcal{E}}_{X / Y}^{(s)}=\left\{P \in \mathcal{E}_{X}^{(s)} ;\left[P, x_{j}\right]=0\right.$ for $\left.1 \leq j \leq n\right\}$ and $\mathcal{E}_{X / Y}^{(s)}:=\mathscr{D}_{X} \tilde{\mathcal{E}}_{X / Y}^{(s)}$. Then integro differential operators associated with $\mathcal{E}_{X / Y}^{(s)}$ act on the space $\tilde{O}^{(s)} / \mathcal{O}_{X, q}$, and the actions are realized as follows (see also Bony-Schapira $[\mathbb{B}-\mathrm{S}]$ and $[\mathbb{K}-\mathbb{K}$; Chapter 3]). For any $P \in \tilde{\mathcal{E}}_{X / Y}^{(s)}$ and $f \in \tilde{O}^{(s)}$,

$$
P_{\lambda} f=P_{0} f+\int_{\lambda}^{t} K\left(x, t, t^{\prime}\right) f\left(t^{\prime}, x\right) d t^{\prime}
$$

where $P_{0} \in \mathscr{D}_{X}^{(s)}$ and $K$ is the holomorphic kernel function associated with $P$. Moreover it is easy to see that for any $\tau \in \pi_{1}(X \backslash Y), P \in \mathcal{E}_{X / Y}^{(s)}$ and $u \in \tilde{O^{(s)}} / \mathcal{O}_{X, q}$, we have

$$
\tau(P u)=P \tau(u)
$$

Now we show if $\mathscr{M}$ has irregularity at most $\sigma$ along $V$ and $\operatorname{supp}(\mathscr{M}) \subset \Lambda$, the multi-valued holomorphic function $\tilde{\phi}(u)$ defined in Theorem 4.2 belongs to $\tilde{O}^{(s)}$.

Proposition 4.3. Let $\phi \in \operatorname{Hom}_{\bar{z}}^{(s)}\left(\mathscr{M}^{(s)}, C\right)$ and $u \in \mathscr{M}_{p}^{(s)}$. If $\mathscr{M}$ has irregularity at most $\sigma$ along $V$ with $\operatorname{supp}(\mathscr{M}) \subset \Lambda$, then any holomorphic representation $\tilde{\phi}(u)$ of $\phi(u)$ belongs to $\tilde{O}^{(s)}$ for all $s \in\left[1, \frac{\sigma}{\sigma-1}\right]$.

Proof. We first consider the case $u \in \mathscr{M}_{p}$. Set $\Re=\mathcal{E}_{X} u$. Then $\mathscr{\Omega}$ has irregularity at most $\sigma$ along $V$ and $\operatorname{supp}(\mathcal{R})$ is contained in $\Lambda$. We need the following lemma.

Lemma 4.4. There exist a differential operator $Q\left(t, x, \partial_{t}\right)$ and micro-differential operators $P_{i}\left(x, t, \partial_{t}, \partial_{x_{i}}\right)(1 \leq i \leq n)$ which satisfy the conditions (1), (2) and (3).

(1) All $Q$ and $P_{i}$ 's annihilate $u$ (i.e. $Q u=P_{i} u=0$ ).

(2) The differential operator $Q$ has the form

$$
Q=t^{d} \partial_{0}^{m_{0}}+\sum_{k=0}^{m_{0}-1} a_{k}(x, t) t^{d_{k}} \partial_{t}^{k}
$$

where $d_{k} \in \mathbb{N}$ satisfies

$$
\max _{k}\left\{\frac{d_{0}-d_{k}}{m_{0}-k}\right\} \leq \sigma
$$


(3) The micro-differential operators $P_{i}$ have the forms

$$
P_{i}=\partial_{x_{i}}^{m_{i}}+\sum_{k=0}^{m_{i}-1} \widetilde{P}_{i, k}\left(x, t, \partial_{t}\right) \partial_{x_{i}}^{k}
$$

where $\widetilde{P}_{i, k}$ are micro-differential operators satisfying $\operatorname{ord}\left(\widetilde{P}_{i, k}\right)<m_{i}-k$ and $\left[\widetilde{P}_{i, k}, x_{j}\right]=0$ for all $1 \leq i, j \leq n, 0 \leq k \leq m_{i}-1$.

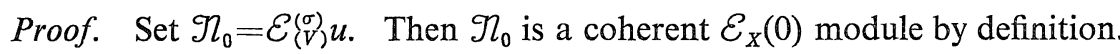
Moreover on account of [B; Lemma of p. 53], $\Re_{0}$ is a finitely generated $\mathcal{O}_{X, q}$ module. By the same argument as Lemma 2.11, we can find a differential operators $Q$. Since $\operatorname{supp}(\mathscr{M})$ is contained in $\Lambda$, we find, for any $i$, an integer $m_{i}$ such that $\partial_{x_{i}}^{m_{i}} \partial_{t}^{-m_{i}+1} u \in \Re_{0}$. Then employing the same argument as Lemma 2.10,

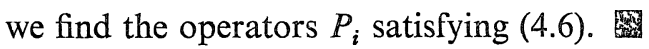

Continue to the proof of Proposition 4.3. Since $Q u=P_{i} u=0$, there exists $\varepsilon>0$ so that

$$
Q \tilde{\phi}(u) \in \mathcal{O}\left(B_{\varepsilon}\right)
$$

By (4.4) and (4.7), it is easy to see $\tilde{\phi}(u)$ is extended over $B_{\varepsilon} \backslash Y$. Moreover on account of the condition (4.5) and Gronwall's inequality, $\tilde{\phi}(u)$ belongs to $\tilde{O}^{(s)}$ for $s \in\left[1, \frac{\sigma}{\sigma-1}\right]$. In general case, let $P \in \mathcal{E}_{X}^{(s)}$. Then dividing $P$ by $P_{1}, \cdots, P_{n}$, we can find the operator $R \in \mathcal{E}_{X / Y}^{(s)}$ such that $P u=R u$. Thus we obtain

$$
\tilde{\phi}(P u)=\tilde{\phi}(R u)=R \tilde{\phi}(u) \quad \bmod \mathcal{O}_{X, q},
$$

and $R \widetilde{\phi}(u) \in \tilde{O}^{(s)}$ by the previous remarks.

Now we enter into the proof of Theorem 4.1.

Proof of Theorem 4.1. Let $c$ be the subset of $\mathbb{C}[r]$ consisting of $\tau \in \mathbb{C}[r]$ with the property that $\tau(\varphi)$ is holomorphic in a neighborhood of $q=\pi(p)$ for any $\phi \in \operatorname{Ham}_{\mathcal{X}}(\mathscr{M}, C)$ and any representative $\varphi$ of any element of $\phi\left(\mathcal{M}_{p}\right)$. Then by (4.2), $c \neq \emptyset$. Moreover for any $\phi \in \operatorname{Ham}_{X}^{(s)}\left(\mathcal{M}^{(s)}, C\right)$, any representative $\tilde{\phi}$ of any element of $\phi\left(\mathscr{M}_{p}^{(s)}\right)$ and $\tau \in c, \tau(\tilde{\phi})$ is holomorphic at $q$ on account of (4.3). Let $\mathcal{L}$ be a holonomic system of $D$ type with monodoromy type $a:=(r-1) c, \mathcal{P}=\operatorname{Im}\left(\mathcal{O}_{q} \otimes_{c} \operatorname{Hom}_{\mathscr{D}_{X}}(\mathcal{O}, \mathcal{L})_{q} \rightarrow \mathcal{L}_{q}\right)$ and $\phi \in \operatorname{Hom}_{X}^{\infty}\left(\mathscr{H}^{\infty}, C\right)$. Set $\Re=\frac{\mathcal{L}}{\mathscr{Q}}$. We define the morphism $E(\phi)$ by 


$$
\begin{aligned}
E(\phi): \mathscr{M}_{p}^{\infty} & \rightarrow \mathscr{N}_{q}^{\infty} \\
u & \rightarrow \widetilde{\phi}(u) \bmod \mathscr{Q} .
\end{aligned}
$$

Since we have $E(\phi)\left(\mathscr{M}_{p}^{(s)}\right) \subset \mathscr{N}_{q}^{(s)}$ by Proposition 3.5 and Proposition 4.3, we obtain the $\mathscr{D}_{X, q}^{(s)}$ morphism $E^{(s)}(\phi): \mathscr{M}_{p}^{(s)} \rightarrow \mathscr{N}_{q}^{(s)}$. Using $E(\phi)$ (resp. $E^{(s)}(\phi)$ ), we can construct the $\mathscr{D}_{X}^{\infty}\left(\right.$ resp. $\left.\mathscr{D}_{X}^{(s)}\right)$ morphism $F(\phi)$ (resp. $\left.F^{(s)}(\phi)\right)$ as follows.

$$
\begin{aligned}
u & \rightarrow 1 \otimes E(\phi)(u) \\
F(\phi): \quad\left(\mathscr{M}^{\infty}\right)_{p} & \rightarrow\left(\mathcal{E}_{X}^{\infty} \otimes \mathcal{D}_{X}^{\infty} \mathcal{I}^{\infty}\right)_{p} \\
\hat{\jmath} & \\
F^{(s)}(\phi): \quad\left(\mathscr{M}^{(s)}\right)_{p} & \rightarrow\left(\mathcal{E}_{X}^{(s)} \otimes \mathscr{D}_{X}^{(s)} \mathscr{I}^{(s)}\right)_{p} \\
u & \rightarrow 1 \otimes E^{(s)}(\phi)(u) .
\end{aligned}
$$

Since $\operatorname{Hom}_{\mathcal{E}_{X}}(\mathscr{M}, C)$ is finite dimensional over $\mathbb{C}$, we can choose a base $\left(\phi_{1}, \cdots, \phi_{r}\right)$ of $\operatorname{Hom}_{\mathcal{X}}(\mathscr{M}, C)$ and obtain the commutative diagram

$$
\begin{aligned}
& \widetilde{F}=F\left(\phi_{1}\right) \oplus \cdots \oplus F\left(\phi_{r}\right): \quad\left(\mathscr{H}^{\infty}\right)_{p} \rightarrow \bigoplus\left(\mathcal{E}_{X}^{\infty} \otimes \mathscr{D}_{X}^{\infty} \mathscr{I}^{\infty}\right)_{p}
\end{aligned}
$$

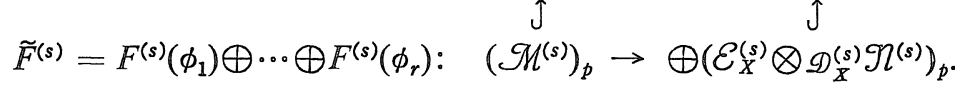

$\widetilde{F}$ is an injective $\mathcal{E}_{X}^{\infty}$ linear morphism by $[\mathbb{K}-\mathbb{K}$; Proposition 4.6.2 and Proposition 4.7.1]. The morphisms of the first and second columns are injective on account of faithful flatness. Thus $\widetilde{F}^{(s)}$ is an injective $\mathcal{E}_{X}^{(s)}$ morphism. This completes the proof of Theorem 4.1.

Using Theorem 4.1 and applying the same argument as $[\mathbb{K}-\mathbb{K}$; Theorem 5.2.1], we obtain the following corollary.

Corollary 4.5. Let $\mathscr{M}$ be a holonomic $\mathcal{E}_{X}$ module at $p$ with $\operatorname{supp}(\mathscr{M}) \subset \Lambda$. We assume $\mathscr{M}$ has irregularity $\sigma$ along $V$. Then there exists a holonomic $\mathcal{E}_{X}$ module $\mathscr{N}$ at $p$ with $R$.S. such that $\mathcal{E}_{X}^{(s)} \otimes \mathcal{E}_{X} \mathscr{M} \simeq \mathcal{E}_{X}^{(s)} \otimes \mathcal{E}_{X} \Re$ for $s \in\left[1, \frac{\sigma}{\sigma-1}\right]$.

Moreover by employing quantized contact transformation, we obtain the following general result.

Corollary 4.6. Let $W$ be a maximally degenerate involutive submanifold of $\stackrel{\circ}{T}^{*} X, \Lambda$ its singular locus and $\mathscr{M}$ a holonomic $\mathcal{E}_{X}$ module with $\operatorname{supp}(\mathscr{M}) \subset \Lambda$. Assume $\mathscr{M}$ has irregularity at most $\sigma$ along $W$. Then there exists a holonomic $\mathcal{E}_{X}$ module $\Re$ at $p$ with $R . S$. such that $\mathcal{E}_{X}^{(s)} \otimes \mathcal{E}_{X} \mathscr{M} \simeq \mathcal{E}_{X}^{(s)} \otimes \mathcal{E}_{X} \Re$ for $s \in\left[1, \frac{\sigma}{\sigma-1}\right]$. 


\section{$\S 5$. Proof of the Main Theorem}

The aim of this section is to prove the main theorem. To obtain the same result as Corollary 4.6 on the singular points of $\operatorname{supp}(\mathscr{M})$, we need the following vanishing theorem.

Theorem 5.1 (cf. [K-K; Theorem 1.2.1]). Let $\mathscr{M}$ be a coherent $\mathcal{E}_{X}$ module and $Z$ a closed analytic subset of $T^{*} X$ (not necessarily homogeneous). Then we have

$$
H_{Z}^{j}\left(\frac{\mathcal{E}_{X}^{(s)} \otimes \mathcal{E}_{X} \mathscr{M}}{\mathcal{E}_{X}^{\left(s^{\prime}\right)} \otimes_{\mathcal{E}_{X}} \mathscr{M}}\right)=0
$$

for $j<\operatorname{codim}_{T^{*} X} Z$-projdim $\mathscr{M}$ and $1 \leq s \leq s^{\prime}<\infty$.

Proof. We use the idea of [K-K; Theorem 1.2.1].

(I) The case where $Z \subset \stackrel{\circ}{T}^{*} X$ and $Z$ is homogeneous. By the induction on projdim $\mathscr{M}$, we may assume $\mathscr{M}$ is a free $\mathcal{E}_{X}$ module. Thus it is sufficient to show

$$
H_{Z}^{j}\left(\frac{\mathcal{E}_{X}^{(s)}}{\mathcal{E}_{X}^{\left(s^{\prime}\right)}}\right)=0 \quad\left(j<\operatorname{codim}_{T^{*} X} Z\right)
$$

Moreover since $\mathcal{E}_{X}^{(s)}=\mathcal{C}_{X \mid X \times X}^{(s)}$, we will show

$$
H_{Z}^{j}\left(\frac{\mathcal{C}_{X \mid X \times X}^{(s)}}{\mathcal{C}_{X \mid X \times X}^{(s \prime)}}\right)=0
$$

for a closed subset $Z \subset \stackrel{\circ}{T}_{X}^{*}(X \times X)$ and $j<\operatorname{codim}_{T^{*} X} Z=\operatorname{codim}_{T_{X}^{*}(X \times X)} Z$. Employing a quantized contact transformation, we may replace $\mathcal{C}_{X \mid X \times X}^{(s)}$ to $\mathcal{C}_{Y \mid X \times X}^{(s)}$ for a nonsingular hypersurface $Y$ of $X \times X$. From now on, we denote $X \times X$ by the same letter $X$. Moreover since $Z$ is homogeneous, (if we regard $\mathcal{C}_{Y \mid X}^{(s)}$ and $\mathcal{C}_{Y \mid X}^{\left(s^{\prime}\right)}$ as sheaves on $Y$ ), it suffices to show

$$
H_{Z}^{j}\left(\frac{\mathcal{C}_{Y}^{(s)}}{\mathcal{C}_{Y \mid X}^{(s)}}\right)=0
$$

for a closed analytic subset $Z$ of $Y$ and $j<\operatorname{codim}_{Y}(Z)$.

Next we shall show that we have only to consider the case that $Z$ is nonsingular. But since the proof of this part goes in the same way as [K-K; Theorem 1.2.1, p. 839], we omit it.

Now we assume $Z$ is nonsingular, and we are in the following situation. 


$$
\begin{aligned}
& X=\mathbb{C} \times \mathbb{C}^{l} \times \mathbb{C}^{d}=(t, y, z), \\
& Y=\{t=0\}, \quad Z=\{t=y=0\} .
\end{aligned}
$$

By the edge of the wedge for the sheaves $\mathcal{C}_{Y \mid X}^{(s)}$ (See [L; Theorem 1.1.4 and p. 47]), we have

$$
H_{Z}^{j}\left(\mathcal{C}_{Y \mid X}^{(s)}\right)=H_{Z}^{j}\left(\mathcal{C}_{Y \mid X}^{\left(s^{\prime}\right)}\right)=0 \quad(j \neq l) .
$$

Considering the long exact sequence

$$
\begin{aligned}
\cdots & \rightarrow H_{Z}^{l-1}\left(\mathcal{C}_{Y \mid X}^{\left(s^{\prime}\right)}\right) \rightarrow H_{Z}^{l-1}\left(\mathcal{C}_{Y \mid X}^{(s)}\right) \rightarrow H_{Z}^{l-1}\left(\frac{\mathcal{C}_{Y \mid X}^{(s)}}{\mathcal{C}_{Y \mid X}^{\left(s^{\prime}\right)}}\right) \\
& \rightarrow H_{Z}^{l}\left(\mathcal{C}_{Y \mid X}^{\left(s^{\prime}\right)}\right) \rightarrow H_{Z}^{l}\left(\mathcal{C}_{Y \mid X}^{(s)}\right) \rightarrow \cdots,
\end{aligned}
$$

we have $H_{Z}^{j}\left(\frac{\mathcal{C}_{Y \mid X}^{(s)}}{\mathcal{C}_{Y \mid X}^{(s)}}\right)=0$ for $j \leq l-2$. To obtain $H_{Z}^{l-1}\left(\frac{\mathcal{C}_{Y}^{(s)}}{\mathcal{C}_{Y \mid X}^{(s)}}\right)=0$, it is enough to show $H_{Z}^{l}\left(\mathcal{C}_{Y \mid X}^{\left(s^{\prime}\right)}\right) \rightarrow H_{X}^{l}\left(\mathcal{C}_{Y \mid X}^{(s)}\right)$ is injective. Set $B_{\varepsilon}=\{w \in \mathbb{C} ;|w|<\varepsilon\}$ and

$$
\begin{aligned}
& T_{\varepsilon}=B_{\varepsilon}-\{0\} \subset \mathbb{C}, \quad U_{\mathrm{\varepsilon}}=B_{\mathrm{\varepsilon}}^{l+d} \subset Y, \quad W_{\mathrm{\varepsilon}}=B_{\mathrm{\varepsilon}}^{d} \subset Z, \\
& V_{\mathrm{\varepsilon}}=T_{\varepsilon}^{l} \times B_{\mathrm{\varepsilon}}^{d} \subset Y, \\
& V_{\mathrm{\varepsilon}}^{j}=T_{\mathrm{\varepsilon}} \times \cdots \times T_{\mathrm{\varepsilon}} \times B_{\mathrm{\varepsilon}} \times T_{\mathrm{q}} \cdots \times T_{\mathrm{q}} \times B_{\mathrm{\varepsilon}}^{d} \quad(1 \leq j \leq l) .
\end{aligned}
$$

For an open set $U$ of $Y$ and $V$ of $Z$, we define two spaces by

$$
\begin{aligned}
S^{(s)}(U)=\{ & \left\{P_{i}(y, z)\right\}_{i \in Z} \in \mathcal{O}(U) ; \forall K \Subset U, \exists C \\
& \left.\left|P_{i}(y, z)\right| \leq \frac{C^{i}}{i !^{s}}, \quad\left|P_{-i}(y, z)\right| \leq C^{i} i ! \quad(i \geq 0)\right\}, \\
\mathcal{H}^{(s)}(V)= & \left\{\left\{P_{i, \alpha_{1}, \cdots, \alpha_{l}}(z)\right\}_{i \in Z, \alpha_{1}>0, \cdots, \infty_{l}>0} \in \mathcal{O}(V) ; \forall K \subset V, \forall \varepsilon>0, \exists C\right. \\
& \left.\left|P_{i, \alpha}(z)\right| \leq \frac{\varepsilon^{|\alpha|} C^{i}}{i !^{s}}, \quad\left|P_{-i, \alpha}(z)\right| \leq \varepsilon^{|\alpha|} C^{i} i ! \quad(i \geq 0)\right\} .
\end{aligned}
$$

Since $V_{\varepsilon}$ and $V_{\varepsilon}^{j}$ are holomorphic domains, we have $H^{i}\left(V_{\varepsilon}, \mathcal{C}_{Y \mid X}^{(s)}\right)=H^{i}\left(V_{\varepsilon}^{j}, \mathcal{C}_{Y \mid X}^{(s)}\right)$ $=0$ for $i>0$ by [L; Theorem 1.1 .3 and p. 47]. Thus we have $\stackrel{V}{C} e c h$ representation

$$
H_{Z}^{l}\left(U_{\varepsilon}, \mathcal{C}_{Y \mid X}^{(s)}\right)=\frac{\mathcal{C}_{Y \mid X}^{(s)}\left(V_{\varepsilon}\right)}{\bigoplus_{j=1}^{l} \mathcal{C}_{Y \mid X}^{(s)}\left(V_{\varepsilon}^{j}\right)}=\frac{S^{(s)}\left(V_{\varepsilon}\right)}{\bigoplus_{j=1}^{l} S^{(s)}\left(V_{\varepsilon}^{j}\right)} .
$$

Moreover we have the morphism

$$
\begin{aligned}
\Phi: \mathcal{H}^{(s)}\left(W_{\varepsilon}\right) & \rightarrow \frac{S^{(s)}\left(V_{\varepsilon}\right)}{\bigoplus_{j=1}^{l} S^{(s)}\left(V_{\varepsilon}^{j}\right)} \\
\left\{P_{i, \alpha}\right\} & \rightarrow\left\{\sum_{\alpha} P_{i, \alpha} y^{-\alpha}\right\}
\end{aligned}
$$


The morphism (5.4) is isomorphic by the property of Laurent expansions. In the same way for $s^{\prime}$, we have the commutative diagram

$$
\begin{array}{ccc}
H_{Z}^{l}\left(V_{\varepsilon}, \mathcal{C}_{Y \mid X}^{\left(s^{\prime}\right)}\right) & \rightarrow & H_{Z}^{l}\left(V_{\varepsilon}, \mathcal{C}_{Y \mid X}^{(s)}\right) \\
\| & & \| \\
\mathcal{H}^{\left(s^{\prime}\right)}\left(W_{\varepsilon}\right) & \rightarrow & \mathcal{H}^{(s)}\left(W_{\varepsilon}\right) .
\end{array}
$$

The morphism of the second row is a natural inclusion. Thus we have proved the injectivity of $H_{Z}^{l}\left(\mathcal{C}_{Y \mid X}^{\left(s^{\prime}\right)}\right) \rightarrow H_{Z}^{l}\left(\mathcal{C}_{Y \mid X}^{(s)}\right)$.

(II) For a general case, using the technique of dummy variable, we can easily reduce it to the case (I) (see the final part of the proof of [K-K; Theorem 1.2.1]).

Remark that we have $H_{Z}^{j}\left(\mathscr{H}^{(s)}\right)=0$ for $j<\operatorname{codim}_{T^{*} X} Z$-projdim $\mathscr{M}$ by the same proof.

As a corollary of this theorem, we have

Corollary 5.2. Let $\Omega$ be an open subset of $T^{*} X$ and $Z$ an analytic subset of $\Omega$ with $\operatorname{codim}_{T^{*} X} Z \geq \operatorname{dim} X+1$. Let $\mathscr{M}$ be a coherent $\mathcal{E}_{X}$ module on $\Omega$ and $u \in \Gamma\left(\Omega, \mathscr{M}^{\infty}\right)$. Assume $u$ belongs to $\mathscr{M}^{(s)}$ outside of $Z$. Then $u \in \Gamma\left(\Omega, \mathscr{M}^{(s)}\right)$. Moreover if $\operatorname{codim}_{T^{*} X} Z \geq \operatorname{dim} X+2$, any section of $\mathscr{M}^{(s)}$ defined on $\Omega-Z$ is uniquely extended to a section of $\mathscr{M}^{(s)}$ defined on $\Omega$.

Now we give the proof of the main theorem.

Proof of the Main Theorem. By [K-K; Theorem 5.2.1], there exist a holonomic module $\mathscr{M}_{\text {reg }}$ with R.S. and an $\mathcal{E}_{X}^{\infty}$ isomorphism $\Phi: \mathscr{M}^{\infty} \rightarrow \mathscr{M}_{r e g}^{\infty}$. Then it is sufficient to show

$$
\Phi\left(\mathscr{H}^{(s)}\right) \subset \mathscr{M}_{\text {reg }}^{(s)} \text { and } \Phi^{-1}\left(\mathscr{M}_{r e g}^{(s)}\right) \subset \mathscr{H}^{(s)} \text {. }
$$

Thereby we will show for holonomic $\mathcal{E}_{X}$ modules $\mathscr{\Omega}_{1}$ and $\mathscr{\Omega}_{2}$ with irregularity at most $\sigma$ and any $\mathcal{E}_{X}^{\infty}$ linear morphism $f: \mathscr{I}_{1}^{\infty} \rightarrow \mathscr{I}_{2}^{\infty}$,

$$
f\left(\mathscr{N}_{1}^{(s)}\right) \subset \mathscr{N}_{2}^{(s)} \quad\left(s \in\left[1, \frac{\sigma}{\sigma-1}\right]\right) .
$$

Let $\Lambda_{1}=\operatorname{supp}\left(\mathscr{\Omega}_{1}\right), \Lambda_{2}=\operatorname{supp}\left(\mathscr{I}_{2}\right)$ and $\Lambda=\Lambda_{1} \cup \Lambda_{2}$. Set $\Lambda_{i r r}=\left(\Lambda_{1}\right)_{i r r} \cup$ $\left(\Lambda_{2}\right)_{i r r}$. First we work at $p \in \Lambda \backslash \Lambda_{i r r}$. If $p \in T$ X $X, \Re_{1}$ and $\mathscr{\Omega}_{2}$ are both de Rham systems. Thus there is nothing to prove. Next we assume $p \notin T_{X}^{*} X$. Then by Corollary 4.6 , there exist holonomic $\mathcal{E}_{X}$ modules $\Re_{1, \text { reg }}, \Re_{2, \text { reg }}$ with R.S. and $\mathcal{E}_{X}^{(s)}$ isomorphisms

$$
\Phi_{1}: \mathscr{\eta}_{1, \text { reg }}^{(s)} \rightarrow \mathcal{N}_{1}^{(s)} \text { and } \Phi_{2}: \mathscr{N}_{2}^{(s)} \rightarrow \mathcal{I}_{2, \text { reg }}^{(s)}
$$


We extend morphisms (5.6) to $\mathcal{E}_{X}^{\infty}$ isomorphisms by $i d \otimes \Phi_{i}(i=1,2)$ and obtain an $\mathcal{E}_{X}^{\infty}$ morphism $\tilde{f}=\left(i d \otimes \Phi_{2}\right) f\left(i d \otimes \Phi_{1}\right): \mathcal{I}_{1, r e g}^{\infty} \rightarrow \mathcal{I}_{2, r e g}^{\infty} . \quad$ By $[\mathbb{K}-\mathbb{K}$; Theorem 6.1.3], we obtain an $\mathcal{E}_{X}$ morphism $f^{\prime}: \mathscr{\Omega}_{1, \text { reg }} \rightarrow \mathscr{\Omega}_{2, \text { reg }}$ such that $\tilde{f}=i d \otimes f^{\prime}$. Thus we have the following commutative diagram:

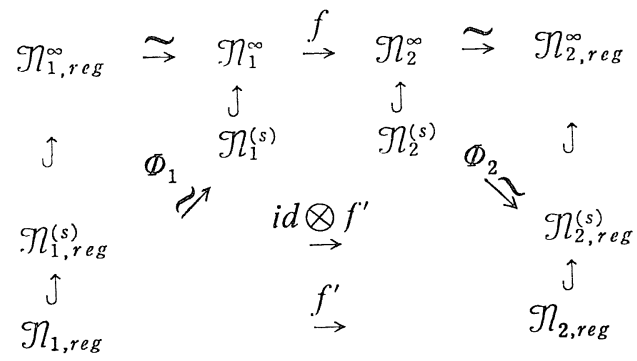

Then easy diagram chasing shows $f\left(\mathcal{I}_{1}^{(s)}\right) \subset \mathscr{I}_{2}^{(s)}$ at $p$. Now we show the claim at $p \in \Lambda_{i r r}$. Let $u \in \mathcal{I}_{1}^{(s)}$. By the above argument, we know $f(u)$ belongs to $\mathcal{T}_{2}^{(s)}$ outside of $\Lambda_{i r r}$. Since $\operatorname{codim}_{T^{*} X}\left(\Lambda_{i r r}\right) \geq \operatorname{dim} X+1$, we obtain $f(u)$ $\in \mathscr{l}_{2}^{(s)}$ on account of Corollary 5.2. This completes the proof. 图

At the end of this section, we give an application of the main theorem. We say a holonomic $\mathcal{E}_{X}$ module $\mathscr{M}$ is in a generic position at $p$ if and only if $\operatorname{supp}(\mathscr{M}) \cap \pi^{-1} \pi(p) \subset \mathbb{C}^{\times} p$.

Theorem 5.3. Let $\mathscr{M}$ be a holonomic $\mathcal{E}_{X}$ module with irregularity at most $\sigma$ at $p$. Assume $\mathscr{M}$ is in a generic position at $p$. Then there exists a holonomic $\mathscr{D}_{X}$ module $\mathscr{F}$ with R.S. at $q=\pi(p)$ such that for $s \in\left[1, \frac{\sigma}{\sigma-1}\right]$,

$$
\mathcal{E}_{X}^{(s)} \otimes \mathcal{E}_{X} \mathscr{H}_{p} \simeq \mathcal{E}_{X}^{(s)} \otimes \mathscr{D}_{X} \mathscr{F}_{q}
$$

Proof. Given a R.S. holonomic $\mathcal{E}_{X}$ module $\mathscr{\Omega}$ with $\operatorname{supp}(\mathscr{R})$ in a generic position. Then by $\left[\mathbb{K}-\mathrm{K}\right.$; Theorem 5.1.4], there exists a holonomic $\mathscr{D}_{X}$ module $\mathscr{F}$ with R.S. such that $\Re_{p}=\mathcal{E}_{X} \otimes \mathscr{D}_{X} \mathscr{F}_{q}$. Thus combing this with the result of the main theorem, we obtain the desired result. 腿

\section{References}

[A1] Aoki, T., Growth order of microdifferential operators of infinite order, J. Fac. Sci. Univ. Tokyo, Sect IA, 29 (1982), 143-159.

[A2] —, An invariant measuring the irregularity of a differential opertor and a microdifferential operator, J. Math. Pures et Appl., 611 (1982), 131-148.

[ B ] Björk, J.E., Some results about holonomic $\mathcal{E}$-modules, Publication de l' Université de Recherche Mathematiques, 33, Univ. Louis-Pasteur, 49-62.

[B-S] Bony, J.M. and Schapira, P., Propagation des singularités analytiques pour les solu- 
tions des équations aux derivées partielles, Ann. Inst. Fourier, Grenoble, 26 (1976), 81-140.

[ K1 ] Kashiwara, M., On the maximally overdetermined systems of linear differential equations, I, Publ. RIMS, Kyoto Univ., 10 (1975), 563-579.

[ $\mathrm{K} 2$ ] Math., 49 (1978), 121-135.

[ K3 ] - The Riemann-Hilbert problem for holonomic systems, Pub!. RIMS, Kyoto Univ., 20 (1984), 319-365.

[K-K] Kashiwara, M. and Kawai, T., On the holonomic systems of microdifferential equations III, Publ. RIMS, Kyoto Univ., 17 (1981), 813-979.

[K-O] Kashiwara, M. and Oshima, T., Systems of differential equations with regular singularities and their boundary value problems, Ann. of Math., 106 (1977), 145-200.

[K-S1] Kashiwara, M. and Schapira, P., Microlocal study of sheaves, Astérisque, 128 (1985).

[K-S2] - Sheaves on manifolds, Grundlehren der Math., 292, Springer-Verlag, 1990.

[Ko1] Komatsu, H., On the regularity of hyperfunction solutions of linear ordinary differential equations with real analytic coefficients, J. Fac. Sci. Univ. Tokyo, Sec. IA, 20 (1973), 107-119.

[ Ko2] - Linear hyperbolic equations with Gevrey coefficients, J. Math. Pure et Appl., 59 (1980), 145-185.

[ L ] Laurent, Y., Théorie de la deuxième microlocalisation dans le domine complexe, Progress in Mathematıcs, 53, Birkhäuser, 1985.

[ M1 ] Malgrange, B., Sur les points singuliers des équations différentielles, Enseignement Math., 20 (1974), 147-176.

[ M2 ] - Modules microdifférentiels et classes de Gevrey, Advance in Math., 7B (1981), 515-530.

[ Me ] Mebkhout, Z., Une équivalence de catégories, Compositio Math., 51 (1984), 51-62.

[S-K-K] Sato, M., Kawai, T. and Kashiwara, M., Hyperfunctions and pseudodifferential equations, Lecture Notes in Math., 287, Springer-Verlag, (1973), 265-529.

[ S ] Schapira, P., Microdifferential systems in the complex domain, Grundlehren der Math., 269, Springer-verlag, 1985. 
